\title{
Postgraduate education on palliative care for general practitioners in Belgium
}

General practitioners (GP) highly depend on postgraduate education to keep up their medical competences during their clinical career. This is even more important when it concerns palliative care competences since there is no mandatory undergraduate curriculum in palliative care in Belgium. Multiple organizations offer postgraduate education to GP's without central management of content or quality.

\section{Questions}

- What is the amount, content and educational method of postgraduate education on palliative care in Belgium in 2007?

- Were the educational activities assessed? If yes, how?

- Is the training adapted to the learning needs of GP's?

\section{Method}

We explored the official national dataset of registered continuing medical education (CME) activities and conducted a questionnaire survey among official CME providers to obtain additional information.

Results

$3 \%$ of postgraduate education for GP's in Belgium concerned palliative care.

Most activities dealt with 'end of life decision making' (35\%) and 'pain and symptom control' (21\%). However, this study revealed large gaps in the content of the offered CME: 'communication with patients in the last phase of their life' and 'teamwork' were the most neglected topics ( $11 \%$ and $5 \%$ respectively).

Concerning didactical principles, more than $80 \%$ of the activities were offered as a lecture. Most of them (73\%) were not evaluated. If some evaluation was done, a satisfaction questionnaire for participants was used. Low attendance levels of GP's were seen especially when the target group was composed multidisciplinary.

Data could not reveal quality criteria for the organized sessions. Providers of CME determined the content without inquiring about the learning needs of GP's.

\section{Reflections}

Despite the efforts made to provide CME, we can question if the courses fit their purpose. First the topics offered were incomplete in comparison with the EAPC curriculum suggestions (1). Especially the absence of the more complex themes like communication skills or teamwork expertise impedes the education of GP's. Second the lack of diversity in didactical methods. The excess of lectures at the expense of interactive educational methods is in sharp contrast with literature revealing the inefficiency of lectures in changing professional behavior and patient health status (2). Third, there is a lack of evaluation of the performed CME activities. Measuring the impact of education on GP's professional behavior should be primordial in order to evaluate the efficiency of the education (3). 
Some suggestions for improvement can be made. First, basic information would be welcomed like providing an easy accessible central data set of national activities in order to prevent a fragmented and insufficient educational landscape. Second, CME providers could benefit from more financial support from the government. Most of the local GP organizations are manned by GP's who have late night meetings (after their clinical practice) mostly on voluntary basis to organize CME sessions for their colleagues. These GP's are not trained nor paid to set up complex educational activities to meet the educational needs of their peers. The speakers (often specialists from the local hospitals) who give the lectures are not well prepared for this task as well.

In addition to these supportive measures, a culture of assessment in different ways must be installed: (a) objective assessment of educational needs can direct the content of postgraduate education. This comprises needs on group level or individual level, evolving towards self directed learning; (b) assessment of the didactical value of the educational activities according to quality criteria, can optimize their efficiency; (c) assessment of the educational impact on professional behavior and patient health status should be performed since this must be the final goal.

To yield a maximum result, this optimized offer should reach a maximum number of GP's. Incentives of different kinds can be suggested but it could be more efficient to arouse interest by starting palliative care education at the undergraduate level.

1. Elsner F. Recommendations of the EAPC For the Development of Postgraduate Curricula Leading to Certification in Palliative Medicine 2008: Available from: <http://www.eapcnet.org/download/forTaskforces/PhysiciansTF/EAPCCurrPostgraduateMedEdu.pdf>

2. Forsetlund L, Bjørndal A, Rashidian A, Jamtvedt G, O'Brien MA, Wolf F, Davis D, OdgaardJensen J, Oxman AD. Continuing education meetings and workshops: effects on professional practice and health care outcomes. Cochrane Database of Systematic Reviews 2009, Issue 2. Art. No.: CD003030. DOI: 10.1002/14651858.CD003030.pub2.

3. Kirkpatrick D. Great Ideas Revisited. Techniques for Evaluating Training Programs. Revisiting Kirkpatrick's Four-Level Model. Training and Development1996;50(1):54-9.

Peter Pype

Palliative Home Care, Roeselare, Belgium

Primary and Interdisciplinary Care, University of Antwerp, Belgium

Johan Wens

Primary and Interdisciplinary Care, University of Antwerp, Belgium

Myriam Deveugele

Department of General Practice and Primary Health Care, Ghent University, Belgium

Ann Stes 
Department of Education, University of Antwerp, Belgium

Bart Van den Eynden

Primary and Interdisciplinary Care, University of Antwerp, Belgium

Corresponding author:

Peter Pype

Gitsbergstraat 66

8830 Gits

Belgium

Email: peter.pype@telenet.be 